\title{
In-Depth Study of the Influence of Host-Framework Flexibility on the Diffusion of Small Gas Molecules in One-Dimensional Zeolitic Pore Systems
}

\author{
N. E. R. Zimmermann, ${ }^{*}, \dagger$ S. Jakobtorweihen,, E. Beerdsen, ${ }^{\S}$ B. Smit, ${ }^{\dagger, \S}$ and F. J. Keil ${ }^{\ddagger}$ \\ Centre Européen de Calcul Atomique et Moléculaire (CECAM), Ecole Normale Supérieure, 46 Allée d'Italie, \\ 69007 Lyon, France, Hamburg University of Technology, Chemical Reaction Engineering, Eissendorfer Str. 38, \\ D-21073 Hamburg, Germany, and Van't Hoff Institute for Molecular Sciences, University of Amsterdam, \\ Nieuwe Achtergracht 166, 1018 WV Amsterdam, The Netherlands.
}

Received: June 15, 2007; In Final Form: August 8, 2007

\begin{abstract}
Molecular-dynamics simulations are performed to understand the role of host-framework flexibility on the diffusion of methane molecules in the one-dimensional pores of AFI-, LTL-, and MTW-type zeolites. In particular, the impact of the choice of the host model is studied. Dynamically corrected Transition State Theory is used to provide insights into the diffusion mechanism on a molecular level. Free-energy barriers and dynamical correction factors can change significantly by introducing lattice flexibility. In order to understand the phenomenon of free-energy barriers reduction, we investigate the motion of the window atoms. The influence that host-framework flexibility exerts on gas diffusion in zeolites is, generally, a complex function of material, host model, and loading such that transferability of conclusions from one zeolite to the other is not guaranteed.
\end{abstract}

\section{Introduction}

Owing to their regular, well-defined structure and vast variety in composition and framework topology, zeolites have attracted the attention of scientists for several decades. They are used in important industrial processes, for example, gas separation, heterogeneous catalysis, or ion exchange. In each of these processes, diffusion plays a decisive role. ${ }^{1}$ Thus, an understanding of the mobility of the molecules under zeolitic confinement is crucial for process-designing purposes. In this respect, molecular simulations have often provided insights that have complemented experimental observations. This is mainly because many microscopic details cannot easily be studied by experimental measurements, as the necessary high resolution of time and length scales of the order of picoseconds and Ångströms are often difficult to achieve.

Barrer and Vaughan ${ }^{2}$ investigated experimentally the permeability of inert gases in zeolite sodalite. Hereby, the diameter of the gas molecules (Lennard-Jones diameter) was larger than the smallest pore diameter. From their results, they concluded that the gas molecules were able to diffuse through the narrow sodalite windows only because the lattice possesses high flexibility. This conjecture was later supported with the help of molecular dynamics (MD) simulations by Deem et al. ${ }^{3}$ and Smirnov and Bougeard. ${ }^{4}$ Both papers provided indications that gas molecules that are even larger than the sodalite windows can diffuse through the pores because of large fluctuations of the window diameter.

Clearly, these results suggest that zeolite flexibility is an important factor that should be taken into account. At present, however, most simulations are carried out with a rigid zeolite, and it is therefore important to quantify the influence of the

\footnotetext{
* Corresponding author. E-mail: nils.zimmermann@cecam.org.

$\dagger$ CECAM.

$\doteqdot$ Hamburg University of Technology.

$\S$ University of Amsterdam.
}

flexibility. In Table 1, we have compiled some of the available literature data (refs 5-12) for methane in various zeolite structures. These data indicate that flexible lattices can enhance the diffusion coefficient by $6-39 \%$. Most of the studies used the model proposed by Demontis et al., ${ }^{13,14}$ as this is a very efficient model for introducing lattice flexibility. However, Leroy et al. ${ }^{12}$ studied the influence of the flexibility model chosen and observed that, for methane in silicalite, there is a substantial impact of how flexibility is modeled.

While the results from the literature show that diffusivities of methane in silicalite are considerably affected by framework flexibility, a flexible framework does not seem to have a strong impact on the diffusive behavior of methane in the onedimensional pores of $\mathrm{AlPO}_{4}-5$; see Table 1. Kantola et al. ${ }^{15}$ and Demontis et al. ${ }^{16}$ showed that flexibility is crucial for the dynamical properties of xenon in $\mathrm{AlPO}_{4}-11$ and of ethane in $\mathrm{AlPO}_{4}-5$. Jakobtorweihen et al. ${ }^{17,18}$ have shown that the diffusion coefficient of adsorbed molecules in the straight channels of carbon nanotubes does not increase but decreases by 2 orders of magnitude at low loading when the motion of the hostlattice atoms is taken into account.

In this work, we examine the flexibility influence and focus on one-dimensional zeolite pores. We want to elucidate in which manner host-framework flexibility affects the diffusion of the gas molecules. For this purpose, we performed extensive equilibrium MD simulations of methane in all-silica AFI-, MTW-, and LTL-type zeolites using both assumptions, rigid and flexible lattices, and two different host force fields.

The remainder of this paper is structured as follows. Section II presents the models and methods that are used. The structures of AFI-, MTW-, and LTL-type zeolites are presented, and the most important computational details are given. In section III, we elucidate some computational particularities and pitfalls that one has to deal with when performing MD simulations in general and in one-dimensional confinements in particular. In section IV, the simulation results are presented. We start with methane 
TABLE 1: Deviations between Self-Diffusion Coefficients Obtained from Flexible- and Rigid-Lattice Simulations of Methane in Different Zeolites at $T \approx 300 \mathrm{~K}^{a}$

\begin{tabular}{rlclc}
\hline & & load & & \multicolumn{1}{c}{$\left(D_{\mathrm{S}}^{\mathrm{FLEX}}-D_{\mathrm{S}}^{\mathrm{RIGID}}\right) /$} \\
ref & zeolite & {$[1 / \mathrm{UC}]$} & \multicolumn{1}{c}{ host model } & $D_{\mathrm{S}}^{\mathrm{RIGID}}[\%]$ \\
\hline 5 & LTA & 1 & Demontis $^{13}$ & 26 \\
6 & Silicalite & 0 & Demontis $^{13}$ & 33 \\
$7^{b}$ & Silicalite & 0.8 & Demontis $^{13}$ & 33 \\
12 & Silicalite & 2 & Demontis $^{13}$ & 26 \\
12 & Silicalite & 4 & Demontis $^{13}$ & 25 \\
12 & Silicalite & 4 & mod. Demontis $^{19}$ & 21 \\
12 & Silicalite & 4 & SGVFF $^{20}$ & 39 \\
8 & Silicalite & 4 & SGVFF $^{20}$ & 31 \\
9 & Silicalite & 5 & Demontis $^{13}$ & 35 \\
12 & Silicalite & 8 & Demontis $^{13}$ & 12 \\
12 & Silicalite & 12 & Demontis $^{13}$ & 33 \\
10 & Silicalite & 12 & Demontis & 26 \\
11 & AlPO & \\
& & 1.5 & harmonic crystal & 6
\end{tabular}

${ }^{a}$ Data taken from literature: refs $5-12 .{ }^{b}$ Note that we took the values for a sorbate diameter of $\sigma_{\mathrm{SS}}=3.5 \AA$ because this one is closest to our methane diameter $\left(\sigma_{\mathrm{CH}_{4}}=3.7 \AA\right)$.

in AFI, followed by a comparison of the influence of flexibility on methane diffusion in the three different zeolites studied here. Finally, we discuss and conclude our results in section V.

\section{Methodology}

A. Host and Guest Models. Zeolites are nanoporous, crystalline solids whose pore sizes are comparable to the diameter of their guest molecules, for example, hydrocarbons and nobel gases. The official nomenclature for identifying a given zeolite topology structure is a three-letter code ${ }^{21}$ which is derived from one of the associated materials that exhibit the topology structure; for example, AFI originates from "aluminophosphate-five" ( $\left.\mathrm{AlPO}_{4}-5\right)$, MTW comes from "Zeolite Socony Mobil-twelve" (ZSM-12), and LTL is from "Linde-type L". Their framework structures are built up by corner-sharing $\mathrm{TO}_{4}$ tetrahedra ${ }^{21}$ where $\mathrm{T}$ stands either for $\mathrm{Al}$ or for $\mathrm{Si}$. These tetrahedra form the pore system, which can be one-, two- or three-dimensional. In addition to the dimensionality, the smoothness of the pores is an important feature for categorizing zeolite structures. Beerdsen et al. suggest to categorize zeolite pores on the basis of differently connected ellipsoids. ${ }^{22}$ They distinguish between cage-type, channel-type, and intersecting channeltype molecular sieves from which the first two classes are important for this work. Cage-type zeolites exhibit large cages connected by narrow windows. These structures pose, in contrast to the channel-type zeolites that have rather smooth pores, large free-energy barriers to diffusing molecules.

In this work, we study siliceous, cation-free versions of the AFI, MTW, and LTL topology structures; see Figure 1. On the basis of the classification of Beerdsen et al. and the cage-towindow ratio $\left(R_{\mathrm{ctw}}\right)$ being a measure for the extent of pore smoothness, it follows that AFI and MTW are smooth channeltype confinements and LTL is a slightly cage-type zeolite. All three structures have in common that their windows, that is, the region where the pore diameter is smallest, consist of rings of 12 oxygen atoms where 2 adjacent $\mathrm{O}$ atoms are connected via the same silicon atom. Table 2 lists the most important data of the three materials. The framework structures were taken from refs $23-25$.

Usually, zeolites are modeled as rigid frameworks in order to investigate adsorption and diffusion phenomena by means of molecular simulations. ${ }^{26-29}$ A rigid framework is often a good approximation, particularly when computing static properties. ${ }^{19}$
However, if one wishes to investigate the influence of hostframework flexibility, models must be chosen that describe the motion of the zeolite atoms. These models are composed of different potential energy terms $U_{i}$ that account for the influence of different physical processes between the atoms of the host system:

$U_{\text {total }}^{\text {host }}=U_{\text {bonds }}+U_{\text {Urey-Bradley }}+U_{\text {angles }}+U_{\text {torsions }}+U_{\mathrm{LJ}}+$
$U_{\text {electrostatics }}$

$U_{\text {total }}^{\text {host }}$ denotes the total potential energy of the host system, $U_{\text {bonds }}$ accounts for $\mathrm{Si}-\mathrm{O}$ bond stretch, $U_{\text {Urey-Bradley }}$ accounts for $\mathrm{Si}-\mathrm{O} / \mathrm{O}-\mathrm{Si}-\mathrm{O}$ or $\mathrm{Si}-\mathrm{O} / \mathrm{Si}-\mathrm{O}-\mathrm{Si}$ bond angle coupling, $U_{\text {angles }}$ accounts for bond angle bend, $U_{\text {torsions }}$ accounts for torsion potential for the $\mathrm{Si}-\mathrm{O}-\mathrm{Si}-\mathrm{O}$ dihedral angle, $U_{\mathrm{LJ}}$ is a LennardJones potential term for nonbonded interactions, and $U_{\text {electrostatics }}$ accounts for nonbonded electrostatic interactions. Two host force fields were used in this work: the modified Demontis model, as proposed by Demontis et al. ${ }^{13}$ and modified by Vlugt and Schenk, ${ }^{19}$ and the force field developed by Nicholas et al. ${ }^{30}$.

The Demontis model is frequently used for computational adsorption and diffusion studies. ${ }^{12,31,32}$ This model considers only interactions between nearest neighbors and is, hence, computationally very efficient. In fact, two simple harmonic potentials make up the whole sum of potential energy terms of the host system; one modeling $\mathrm{Si}-\mathrm{O}$ bond stretch and one (Urey-Bradley term) for $\mathrm{Si}-\mathrm{O} / \mathrm{O}-\mathrm{Si}-\mathrm{O}$ bond angle coupling:

$$
\begin{gathered}
U_{\text {bonds }}=\frac{1}{2} k_{\text {bonds }}\left(r_{\mathrm{Si}-\mathrm{O}}-r_{\mathrm{Si}-\mathrm{O}}^{\mathrm{eq}}\right)^{2} \\
U_{\text {Urey-Bradley }}=\frac{1}{2} k_{\text {Urey-Bradley }}\left(r_{\mathrm{O}-\mathrm{O}}-r_{\mathrm{O}-\mathrm{O}}^{\mathrm{eq}}\right)^{2}
\end{gathered}
$$

Here, $k_{\text {bonds }}$ and $k_{\text {Urey-Bradley }}$ are the potential constants, $r_{\mathrm{Si}-\mathrm{O}}$ and $r_{\mathrm{O}-\mathrm{O}}$ are the instantaneous distances, and $r_{\mathrm{Si}-\mathrm{O}}^{\mathrm{eq}}$ and $r_{\mathrm{O}-\mathrm{O}}^{\mathrm{eq}}$ are the corresponding equilibrium distances, where $\mathrm{O}-\mathrm{O}$ represents two adjacent oxygen atoms that are bonded to the same silicon atom. In contrast to the original model, the modified version ${ }^{19}$ incorporates individual equilibrium distances for the interaction potentials that are taken from the crystal structure; that is, $r_{\mathrm{Si}-\mathrm{O}}^{\mathrm{eq}}$ and $r_{\mathrm{O}-\mathrm{O}}^{\mathrm{eq}}$ are different for different pairs of atoms. Thereby, the modified model reproduces the crystallographic positions of the zeolite atoms accurately. ${ }^{19}$ It has to be pointed out that, in general, crystallographic data of zeolites are obtained at very low temperatures, and therefore, it is not guaranteed that, for the structures studied here, we deal with the true phase at the temperature studied $(300 \mathrm{~K})$. For example, MFI is known to undergo a phase transition with increasing $T$, from orthorhomic to monoclinic. ${ }^{33}$ However, we use the "zero Kelvin" structure also for rigid-lattice simulations.

The Nicholas force field is a general valence force field (GVFF). In addition to a $\mathrm{Si}-\mathrm{O}$ bond stretch and a $\mathrm{Si}-\mathrm{O} / \mathrm{Si}-$ $\mathrm{O}-\mathrm{Si}$ bond angle coupling term, a bond angle bend term (harmonic potential) and a torsion potential (periodic function with a threefold barrier) are considered:

$$
\begin{gathered}
U_{\text {angles }}=\frac{1}{2} k_{\text {angles }}\left(\gamma_{\mathrm{O}-\mathrm{Si}-\mathrm{O}}-\gamma_{\mathrm{O}-\mathrm{Si}-\mathrm{O}}^{\mathrm{eq}}\right)^{2} \\
U_{\text {torsions }}=\frac{1}{2} k_{\text {torsions }}(1+\cos (3 \Phi)) \cdot \mathrm{S}\left(\gamma_{\mathrm{Si}-\mathrm{O}-\mathrm{Si}}\right)
\end{gathered}
$$

where $k_{\text {angles }}$ and $k_{\text {torsions }}$ denote the respective potential constants, $\gamma_{\mathrm{O}-\mathrm{Si}-\mathrm{O}}$ and $\gamma_{\mathrm{Si}-\mathrm{O}-\mathrm{Si}}$ are the $\mathrm{O}-\mathrm{Si}-\mathrm{O}$ and the $\mathrm{Si}-\mathrm{O}-\mathrm{Si}$ angle, 
AFI

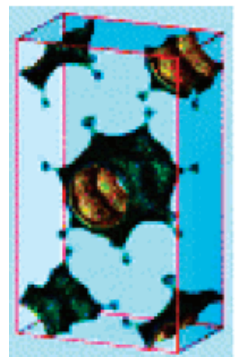

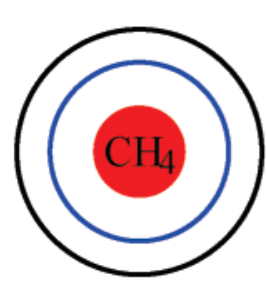

MTW

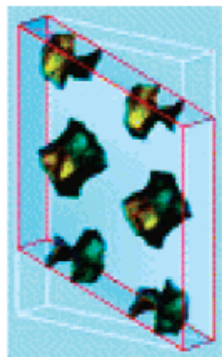

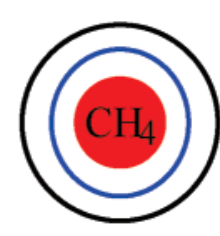

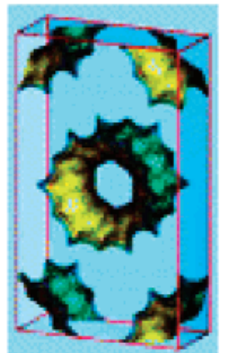

LTL

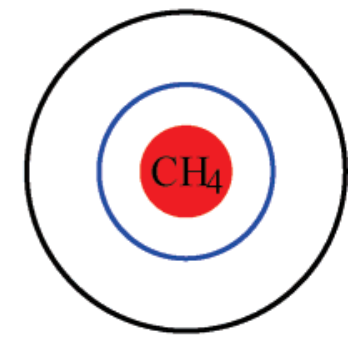

Figure 1. Pore structure of AFI (left), MTW (middle), and LTL (right) zeolite types. In addition, we present schematic drawings that reflect the relation between methane molecule size (Lennard-Jones diameter, filled circles) and the diameters of the cage (outer circles) and the window (inner circle) of the corresponding material.

TABLE 2: Data of the Zeolites Studied

\begin{tabular}{lcccccc}
\hline & window diameter $[\AA]$ & cage diameter $[\AA]$ & $R_{\text {ctw }}$ & unit cell dimension $[\AA](\mathrm{a} \times \mathrm{b} \times \mathrm{c})$ & unit-cell type & simulation box \\
\hline LTL & 7.1 & 13 & 1.83 & $(31.984 \times 18.466 \times 7.474)$ & orthorhombic \\
MTW & 5.6 & 8 & 1.43 & $(24.863 \times 5.012 \times 24.326)$ & monoclinic, $\beta=107.7^{\circ}$ & $2 \times 2 \times 6 \times 2$ \\
AFI & 7.3 & 10 & 1.37 & $(23.774 \times 13.726 \times 8.484)$ & orthorhombic
\end{tabular}

respectively, and $\Phi$ is the dihedral angle. The authors reported that it was necessary to couple the torsion potential to a switching function $\mathrm{S}\left(\gamma_{\mathrm{Si}-\mathrm{O}-\mathrm{Si}}\right)$ in order to avoid a discontinuity in the potential as the angle becomes close to linear and inverts from $0^{\circ}$ to $180^{\circ}$. This function is defined by:

$$
\begin{aligned}
& \mathrm{S}\left(\gamma_{\mathrm{Si}-\mathrm{O}-\mathrm{Si}}\right)= \\
& \begin{cases}\frac{\left(\gamma_{\mathrm{off}}-\gamma_{\mathrm{Si}-\mathrm{O}-\mathrm{Si}}\right)^{2}\left(\gamma_{\text {off }}+2 \gamma_{\mathrm{Si}-\mathrm{O}-\mathrm{Si}}-3 \gamma_{\mathrm{on}}\right)}{\left(\gamma_{\text {off }}-\gamma_{\mathrm{on}}\right)^{3}} & \text { if } \gamma_{\mathrm{on}} \leq \gamma_{\mathrm{Si}-\mathrm{O}-\mathrm{Si}} \leq \gamma_{\text {off }} \\
1 & \text { if } \gamma_{\mathrm{Si}-\mathrm{O}-\mathrm{Si}}<\gamma_{\mathrm{on}}\end{cases}
\end{aligned}
$$

where $\gamma_{\text {on }}=170^{\circ}$ and $\gamma_{\text {off }}=180^{\circ}$. Note that we do not consider the nonbonded terms, as these are shown to be of less importance than the other contributions. ${ }^{30}$

Since the two force fields were developed for other zeolite structures than the ones studied in this work, it is not straightforward to judge how well they describe the motion of the frameworks investigated here. Both models have their own specific advantages. While the modified Demontis model is fast and will, irrespective of the zeolite studied, yield average atom positions that are in good agreement with the crystallographic structure, there is evidence that GVFFs lead to more realistic dynamics of zeolites. The latter is because a simplified GVFF was proven to yield better agreement with experimental infrared and Raman spectra than with the Demontis model. ${ }^{20}$

For modeling the guest-guest and guest-host interactions, the choice of a united-atom (UA) model seems to be an optimal compromise. $^{28,29}$ This is, because adsorption in cation-free zeolites takes place at specific sites with little or no electric field. ${ }^{28,29}$ Moreover, although internal degrees of freedom of the fluid molecule can influence its diffusive behavior, ${ }^{9,34}$ the use of a UA model for methane diffusing in the one-dimensional pores of $\mathrm{AlPO}_{4}-5$ was validated by Fritzsche et al. ${ }^{35}$ They showed that there is no coupling between rotational and translational motion of the gas molecules. Furthermore, we assume for the guest-host interaction that the large oxygen atoms of the solid shield the smaller silicon atoms and, therefore, dominate the guest-host interaction. ${ }^{36}$ Effectively, the $\mathrm{CH}_{4}-$ $\mathrm{Si}$ interactions are accounted for by the $\mathrm{CH}_{4}-\mathrm{O}$ interactions. We chose the force field of Dubbeldam et al. ${ }^{28,29}$ because it was developed to reproduce specifically hydrocarbon adsorption isotherms in zeolites and matches, for instance, the inflection point and maximum loading of methane in $\mathrm{AlPO}_{4}-5$ at $77 \mathrm{~K}$
TABLE 3: Potential Parameters for Host-Host,

\begin{tabular}{|c|c|}
\hline $\begin{array}{l}\text { SYSTEM } \\
\text { force field }\end{array}$ & parameters \\
\hline $\begin{array}{l}\text { HOST-HOST } \\
\text { mod. Demontis }{ }^{19}\end{array}$ & $\begin{array}{l}k_{\text {bonds }} / k_{\mathrm{B}}=251,778 \mathrm{~K} / \AA^{2} \\
r_{\text {bonds }}^{\text {eq }} \text { from crystal structure } \\
k_{\text {Urey-Bradley }} / k_{\mathrm{B}}=51,866 \mathrm{~K} / \AA^{2} \\
r_{\text {Urey-Bradley }}^{\text {re }} \text { from crystal structure }\end{array}$ \\
\hline $\begin{array}{l}\text { HOST-HOST } \\
\text { Nicholas }^{30}\end{array}$ & 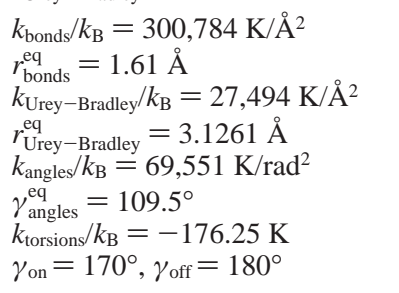 \\
\hline $\begin{array}{l}\text { HOST-GUEST } \\
\text { Dubbeldam }{ }^{28,29}\end{array}$ & $\begin{array}{l}\epsilon_{\mathrm{LJ}, \mathrm{O}-\mathrm{CH}_{4}} / k_{\mathrm{B}}=115.0 \mathrm{~K} \\
\sigma_{\mathrm{LJ}, \mathrm{O}-\mathrm{CH}_{4}}=3.47 \AA\end{array}$ \\
\hline $\begin{array}{l}\text { GUEST-GUEST } \\
\text { Dubbeldam }^{28,29}\end{array}$ & $\begin{array}{l}\epsilon_{\mathrm{LJ}, \mathrm{CH}_{4}-\mathrm{CH}_{4}} / k_{\mathrm{B}}=158.5 \mathrm{~K} \\
\sigma_{\mathrm{LJ}, \mathrm{CH}_{4}-\mathrm{CH}_{4}}=3.72 \AA\end{array}$ \\
\hline
\end{tabular}
Host-Guest, and Guest-Guest Systems

correctly. ${ }^{29}$ Following Dubbeldam et al., the cutoff radius was set to $12 \AA$, and the potential was truncated and shifted, because analytical tail-corrections do not apply in zeolites. ${ }^{29,37}$ In Table 3 , the potential parameters of all models used in this work are summarized. Finally, note that the adsorption of methane in AFI-, LTL-, and MTW-type zeolites has been studied experimentally (refs 38 and 39) and by means of molecular simulations (refs 40-44).

B. Diffusion. Self or tracer diffusion is related to the diffusive motion of a single tagged particle moving around other particles. The quantity associated with this type of diffusion, the selfdiffusion coefficient $D_{\mathrm{S}}$, is, hence, a particle property. $D_{\mathrm{S}}$ can be computed easily by means of MD simulations sampling $\left[\mathbf{r}_{i}(t)-\mathbf{r}_{i}(0)\right]^{2}$, the mean-squared displacement (MSD), of a single tagged particle $i$ as a function of time. The Einstein equation yields for normal-mode diffusion along a onedimensional coordinate $r_{\alpha}$ :

$$
D_{\mathrm{S}, \alpha}=\frac{1}{2 N} \cdot \lim _{t \rightarrow \infty} \frac{\mathrm{d}}{\mathrm{d} t}\left\langle\sum_{i=1}^{N}\left[r_{i, \alpha}(t)-r_{i, \alpha}(0)\right]^{2}\right\rangle
$$

where $N$ denotes the number of particles, $t$ is the time, $\alpha$ is one of the Cartesian coordinates $x, y$, or $z$, and the angular brackets 


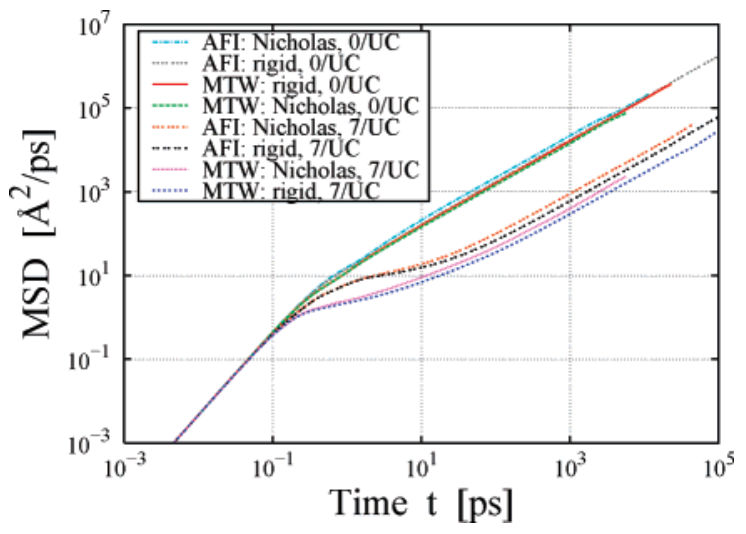

Figure 2. Mean-squared displacement, MSD, as a function of time, $t$, for $\mathrm{CH}_{4}$ in MTW and AFI at $300 \mathrm{~K}$ for different loadings and different frameworks.

indicate an ensemble average. Subsequently, we obtain for the three-dimensional, macroscopic observable diffusivity $D_{\mathrm{S}}$ :

$$
D_{\mathrm{S}}=\frac{D_{\mathrm{S}, x}+D_{\mathrm{S}, y}+D_{\mathrm{S}, z}}{3}
$$

It is important to stress that evaluating the MSD correctly is equivalent to fitting the data to a linear function in the diffusive regime where the MSD is proportional to $t$. Therefore, one has to be aware, first, of the initial ballistic regime ${ }^{45}$ where the MSD is proportional to $t^{2}$ and, second, of a transition regime. The lengths of both regimes are mainly influenced by the actual combination of host and guest and by the system temperature. The transition regime is additionally dependent on loading; ${ }^{12,29}$ see also Figure 2. Both issues are covered in eq 7 by taking the slope at very long times. A double-logarithmic plot, as displayed in Figure 2, is a good means to find the diffusive regime (logarithmic slope of MSD is equal to unity). We have to stress that single-file behavior $\left(D_{\mathrm{S}} \propto \sqrt{t}\right)$ is not observed for any of the systems studied here.

The MSDs are sampled using an order- $n$ algorithm, as it reduces memory requirements and CPU time. ${ }^{46}$ At least three independent simulations are performed for every diffusivity presented in this work in order to give an appropriate error estimate. From this perspective, most of the data of Table 1 should be interpreted with care. In some cases, we could not estimate the accuracy of the data given. In other cases, the errors presented were based on the deviation of the fitted function from the MSD data. On the basis of our analysis, this gives a too small error estimate in comparison with the error obtained by performing independent simulations. Even block averaging may still yield a too small estimate because it is sampled in a too correlated region of phase space.

For obtaining correct values of $D_{\mathrm{S}}$, one has to study long pores due to a strong system size effect that occurs in onedimensional confinements. ${ }^{47} \mathrm{We}$ study, however, relatively small systems for comparing rigid and flexible results. The reasons are discussed in section III.

C. Dynamically Corrected Transition State Theory. As has been shown previously, ${ }^{22,26,27,48,49}$ dynamically corrected Transition State Theory (dcTST) can describe the diffusion of molecules under zeolitic confinement correctly and provide the link between diffusion coefficient and free-energy barriers. In dcTST, diffusion processes in confinements are pictured as hopping events on a lattice from state (lattice site) A to state (lattice site) B. A and B are separated by a lattice distance $\lambda$, and the hopping is limited by a free-energy barrier. ${ }^{22}$ The selfdiffusion coefficient is related to the hopping rate from $\mathrm{A}$ to $\mathrm{B}$ $\left(k_{\mathrm{A} \rightarrow \mathrm{B}}\right.$ in events per unit of time) and $\lambda$ via:

$$
D_{\mathrm{S}}=k_{\mathrm{A} \rightarrow \mathrm{B}} \cdot \lambda^{2}
$$

The hopping rate is determined by:

$$
k_{\mathrm{A} \rightarrow \mathrm{B}}=\kappa \cdot \sqrt{\frac{k_{\mathrm{B}} T}{2 \pi m}} \cdot P\left(q^{*}\right)
$$

where $\kappa$ denotes the transmission coefficient, $k_{\mathrm{B}}$ is Boltzmann's constant, $T$ is the temperature of the system, $m$ is the mass of the hopping particle, and $P\left(q^{*}\right)$ denotes the probability, given that the particle is somewhere in cage A, of observing the transition state (particle on top of the barrier). This probability is obtained from a free-energy profile $F(q)$ :

$$
P\left(q^{*}\right)=\frac{\exp \left[-\beta F\left(q^{*}\right)\right]}{\int_{\text {cage } A} \exp [-\beta F(q)] \mathrm{d} q}
$$

where $q^{*}$ denotes the (assumed) location of the barrier. Finally, we can rewrite and summarize eq 9 to

$$
\begin{aligned}
D_{\mathrm{S}}=\kappa \cdot \sqrt{\frac{k_{\mathrm{B}} T}{2 \pi m}} \cdot P\left(q^{*}\right) \cdot \lambda^{2} \\
=\kappa \cdot D_{\mathrm{S}}^{\mathrm{TST}}
\end{aligned}
$$

$D_{\mathrm{S}}^{\mathrm{TST}}$ represents the ideal TST diffusivity that is based on the assumption that every diffusing particle that comes from cage A and reaches $q^{*}$ ends up successfully in B if it has a velocity that points to $\mathrm{B}$.

By means of eq 13, two different effects can be distinguished. Because of its relation to free-energy barriers which are, for small, uncharged molecules such as the methane molecules in this study, mainly entropic in nature, $D_{\mathrm{S}}^{\mathrm{TST}}$ gives information about the influence of the confinement topology on the diffusion of the gas molecule. On the other side, $\kappa$, also known as the dynamical correction factor, is regarded as a measure for the interaction between the fluid particles themselves. In onedimensional zeolite pores, $\kappa$ is close to unity at infinite dilution, if $q^{*}$ is chosen as the location of the barrier. This is simply because there are no other gas particles present with which the single particle can interact, and a particle crossing the barrier from A to B will usually end up in B. However, once the particles start to interact with one another, $\kappa$ decreases quickly as a function of loading, and Beerdsen et al., ${ }^{22}$ who have studied methane diffusion in several rigid zeolite structures, showed that, even at zero loading, the dynamical correction is nonnegligible (for AFI $\kappa$ is about 0.6). The latter is an interesting observation and shows that the reaction coordinate which is usually a function of the whole configuration space, that is, $q=f\left(\mathbf{r}_{1}, \mathbf{r}_{2}, \ldots, \mathbf{r}_{N}\right)$, is not perfect. Thus, there exist relevant zeolite atoms that play even a role in defining the dividing surface in rigid zeolite structures. However, $\kappa$ was still large in the work of Beerdsen et al. ${ }^{22}$ and the detection of a perfect reaction coordinate that includes the position of relevant zeolite atoms is not straightforward.

For computing free-energy profiles, the histogram sampling (HS) method is used. Since the reaction coordinate $q$ follows from the topology of the structure such that it is simply the 


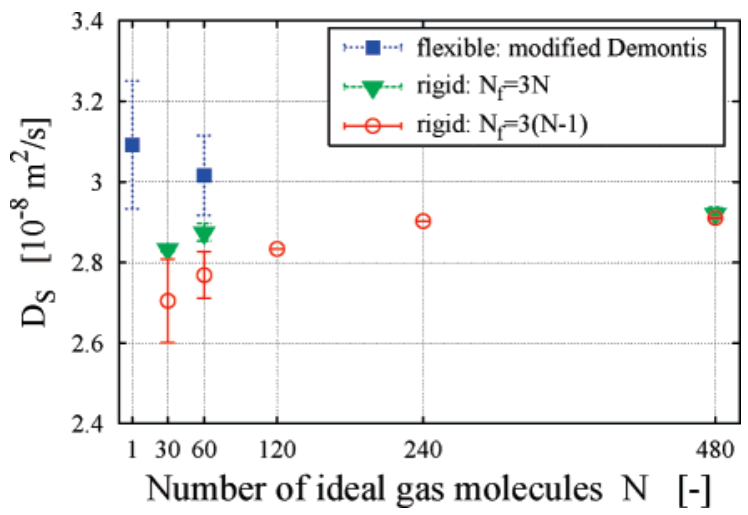

Figure 3. Self-diffusion coefficient, $D_{\mathrm{S}}$, as a function of the number of ideal gas particles, $N$, for $\mathrm{CH}_{4}$ in AFI at $300 \mathrm{~K}$.

progress of a tagged molecule along the Cartesian connection from one cage to another, the free energy is given by:

$$
\beta F(q)=-\ln \langle P(q)\rangle
$$

$P(q)$ denotes the probability to find a molecule at a given value of $q$, that is, at a given position between two adjacent cages. For more details about dcTST, the reader is referred to ref 27.

D. Computational Details. To integrate Newton's equations of motion, we use the velocity Verlet algorithm. ${ }^{46}$ The time step of the numerical integration is set to 2 and 0.5 fs for rigid- and flexible-framework simulations, respectively. Periodic boundary conditions are applied. A Nosé-Hoover chain (NHC) thermostat $^{50}$ is used to control the temperature which is always set to $300 \mathrm{~K}$. The thermostat is coupled, either to the gas molecules (rigid lattice) or to the framework atoms (flexible framework). Thus, the gas molecules are thermalized exclusively via collisions with the vibrating wall atoms during flexible-framework simulations. Our analysis shows that the temperatures of the gas molecules in flexible-lattice simulations is $300 \mathrm{~K}$ on an average; that is, the zeolite atoms thermalize the gas molecules correctly.

\section{Computational Particularities}

A. Limit of Infinite Dilution. Infinite dilution is important as in this limit the self-diffusion coefficient is equal to the transport-diffusion coefficient. Theoretically, one would insert a single particle for simulating at the limit of infinite dilution. However, it is common practice to insert several ideal, that is, noninteracting, molecules for improving statistics. ${ }^{18,27}$ In rigidlattice simulations, this insertion of ideal gas molecules may, however, not be without pitfalls, and also in flexible-lattice simulations, one has to be careful with doing so.

In an NVT simulation, care has to be taken when choosing the number of degrees of freedom $\left(N_{\mathrm{f}}\right)$ for computing the temperature. If one assumes that momentum is conserved (rigid framework), the number of degrees of freedom is $N_{\mathrm{f}}=3(N-$ 1 ), where $N$ is the number of atoms. However, a simulation at infinite dilution is often mimicked with a set of ideal particles. As these ideal particles, by definition, do not interact with one another, the momentum of each particle viewed individually is not fixed; hence $N_{\mathrm{f}}=3 N$. Since $N_{\mathrm{f}}$ is needed for the NHC thermostat for rescaling the velocities, too small an $N_{\mathrm{f}}$ will yield an average temperature that is underestimated on the basis of the true $N_{\mathrm{f}}$. Gas diffusion in zeolites is an activated process that follows the Arrhenius law in most cases such that too low a temperature will result in a too small diffusion coefficient. In fact, that is what we observe; see Figure 3. The diffusivity
TABLE 4: Self-Diffusion Coefficients for Different System Sizes $\left(\mathrm{CH}_{4}\right.$ in AFI, $\left.\theta=1 / \mathrm{UC}, 300 \mathrm{~K}\right)$; In AFI the Pore Direction Runs along $z$

\begin{tabular}{ccccc}
\hline$N_{x}^{\text {unit cell }}[-]$ & $\mathrm{N}_{\mathrm{y}}^{\text {unit cell }}[-]$ & $N_{z}^{\text {unit cell }}[-]$ & $N^{\mathrm{CH}_{4}[-]}$ & $D_{\mathrm{S}}\left[10^{-8} \mathrm{~m}^{2} / \mathrm{s}\right]$ \\
\hline 2 & 3 & 5 & 30 & 1.07 \\
6 & 8 & 5 & 240 & 1.05 \\
2 & 3 & 11 & 66 & 0.78 \\
4 & 6 & 10 & 240 & 0.79 \\
2 & 3 & 40 & 240 & 0.58
\end{tabular}

computed increases much less if $N_{\mathrm{f}}=3 N$. Nonetheless, there is obviously an influence of the system size on $D_{\mathrm{S}}$. For this reason, we performed all zero loading simulations in rigid zeolites with a sufficiently large number of ideal molecules, namely, 480.

For flexible simulations, the limit of infinite dilution poses the following problem when including several ideal gas particles. Since the ideal gas molecules do not interact with one another but do interact with the vibrating wall atoms, there might happen to be an indirect interaction between the gas molecules via the interaction with the wall atoms. To see if this matters for such a long-scale property as the self-diffusion coefficient, we performed two sets of zero-loading simulations with the modified Demontis model:

1. Twenty independent simulations where 1 gas particle is included only. The averaged diffusivity is $3.09( \pm 0.18) 10^{-8}$ $\mathrm{m}^{2} / \mathrm{s}$.

2. Five simulations with 64 ideal methane molecules. $D_{\mathrm{S}}=$ $3.00( \pm 0.13) 10^{-8} \mathrm{~m}^{2} / \mathrm{s}$.

The results indicate that the effect of these indirect interactions on the diffusivity is small, and we obtain better statistics with a shorter computation time; see also Figure 3. Our findings are in agreement to those of Demontis et al. ${ }^{6}$ They investigated the dynamics of ideal methane molecules in a rigid and flexible silicalite framework, respectively. On the basis of methaneframework-oxygen radial distribution functions for rigid and flexible lattices that show same peak locations, they argue that there is no indirect interaction. Note furthermore that our ratio of ideal methane molecules to simulation-box volume is always much smaller than theirs. Thus, we perform zero-loading simulations in flexible frameworks by inserting 60 to 64 ideal gas particles.

B. System-Size Effect. When the gas molecules start to interact with one another, we observe a very strong influence of channel length on the dynamics of the gas molecules. This effect has already been reported by Tepper et al. ${ }^{47}$ who studied methane diffusion in $\mathrm{AlPO}_{4}-5$ for a loading of $0.7 /$ unit cell (UC). They showed that very long pores have to be studied in order to obtain correct dynamic behavior of the gas molecules. We investigate this influence systematically for different loadings $(1 / \mathrm{UC} \leq \theta \leq 7 / \mathrm{UC})$ using the rigid-lattice assumption. For preserving a given loading, channel length $\left(N_{z}^{\text {unitcell }}\right)$ and number of sorbed particles $\left(N^{\mathrm{CH}_{4}}\right)$ are increased proportionally. Scaling of the systems in $x$ and $y$ direction (cross section plane for diffusing molecules) does not affect the diffusivity; see Table 4. The range in these dimensions is only limited by the cutoff radius; in order to avoid self-correlation effects, the dimensions of the simulation boxes have to be at least twice the cutoff radius because, otherwise, particles would interact with themselves (compare the minimum image convention for periodic boundary conditions).

The results of the influence of the system size effect for $\mathrm{CH}_{4}$ in rigid AFI are shown in Figure 4. As a general observation, 


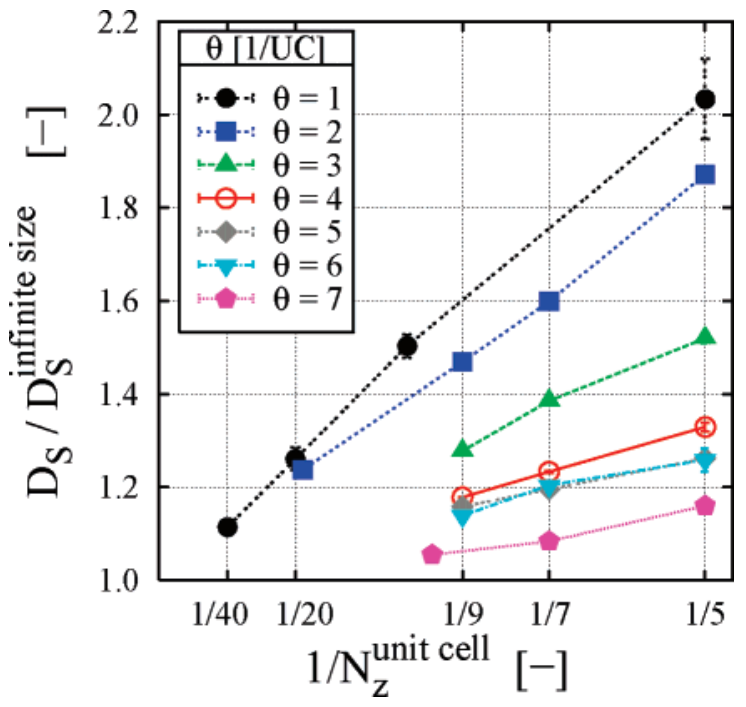

Figure 4. Dependence of inverse channel length, as expressed as the inverse number of unit cells $\left(1 / N_{z}^{\text {unitcell }}\right)$, on the diffusion coefficient, $D_{\mathrm{S}}$, for different unit-cell loadings, $\theta ; \mathrm{CH}_{4}$ in a rigid AFI framework at $300 \mathrm{~K}$. The lines shall guide the eye.

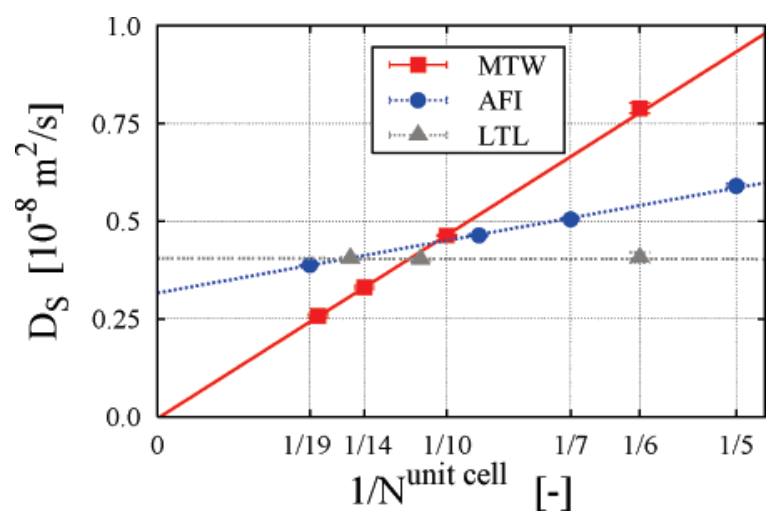

Figure 5. Self-diffusion coefficient, $D_{\mathrm{S}}$, as a function of the inverse channel length, as expressed as the inverse number of unit cells, 1/ $N_{z}^{\text {unitcell }}$; methane in MTW, AFI, and LTL at constant loading of 2/UC and $300 \mathrm{~K}$.

we found that the diffusivities associated with a given loading exhibit a linear dependence on the inverse of channel length. We make use of this fact and determine $D_{\mathrm{S}}$ at infinite system size by extrapolating to $1 / N_{z}^{\text {unitcell }} \rightarrow 0$ and refer to them as $D_{\mathrm{S}}^{\text {infinitesize }}$. Although we study systems that include as many as 40 unit cells in the pore direction, almost all finite systemsize diffusivities are still $10 \%$ larger than the corresponding $D_{\mathrm{S}}^{\text {infinitesize }}$.

Figure 5 shows that this system-size effect is also observed for MTW, where it is even more pronounced, but is not observed for LTL. The negative value of $D_{\mathrm{S}}^{\text {infinitesize }}$ for MTW suggests that the linear dependence will level out at a certain channel length. The influence of channel length on self-diffusivity is also observable for flexible AFI frameworks.

The TST diffusivity, $D^{\text {TST }}$, does not change as a function of channel length for either of the materials studied here. Only the dynamical correction factor, $\kappa$, is affected by the system size (for AFI and MTW). This is comprehensible because $\kappa$ accounts for the number of interparticle collisions which can, of course, change if the channels are elongated. To make this point more clear, consider the situation for AFI with a methane loading of $2 / \mathrm{UC}$; there is thus one molecule per channel and unit cell. If $N_{z}^{\text {unitcell }}$ was chosen to be 1 , there would be no other particles that might induce recrossings; hence, $\kappa$ will be close

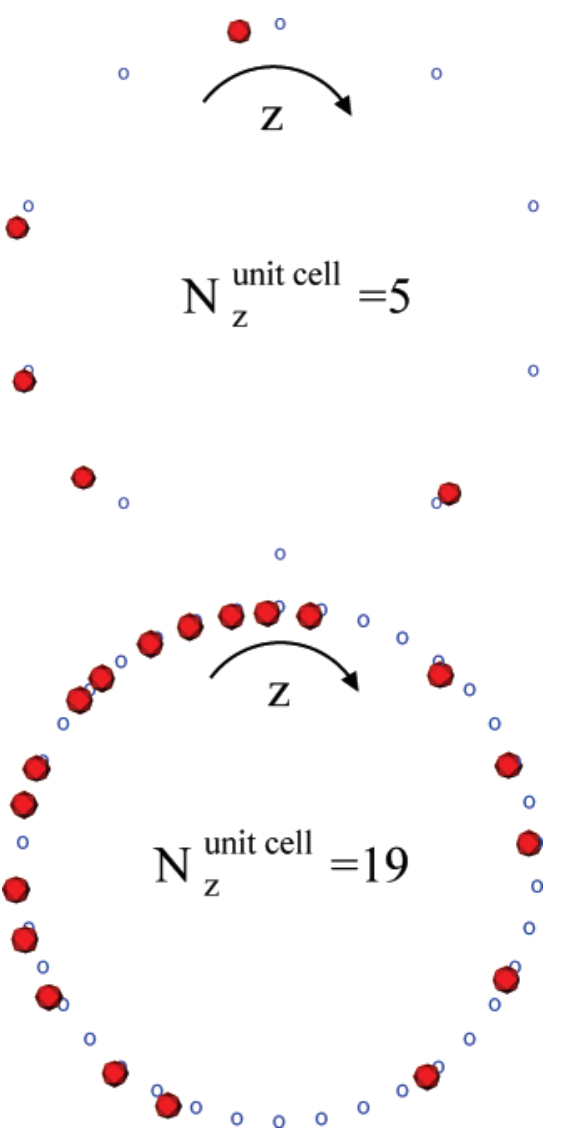

Figure 6. Typical snapshots of the adsorption behavior of methane in AFI (rigid lattice) for $N_{z}^{\text {unitcell }}=5$ and 19; $\theta=2 / \mathrm{UC}, T=300 \mathrm{~K}$. The channel axis is bent to a ring which reflects the use of periodic boundary conditions. The large filled circles represent the methane molecules; the small circles represent the locations of the cages along $z$.

to unity. Clearly, as soon as the channel is elongated, the situation changes and recrossings will be observable; hence, $\kappa^{\text {infinite size }}$ will be smaller than $\kappa\left(N_{z}^{\text {unitcell }}=1\right)$. The snapshots in Figure 6 that show the typical adsorption behavior of methane along the channel axis, $z$, show that the probability of observing an occupied cage that is surrounded by two cages that are also occupied increases with channel length. Hence, the probablility of observing recrossing events that are due to occupied adjacent cages does increase as well. This explanation is consistent with the observation for LTL and MTW when one takes into account the different dimensions of the cages. In LTL, the cages possess so much accessible volume for the methane particles that, at low loadings, it does not matter whether the next cage is occupied or not. The MTW cages, in turn, are smaller than those of AFI such that this effect is even more pronounced here.

C. Lowe-Andersen Thermostat for Interface Fluid Collisions. It is instructive to discuss the effect of zeolite flexibility in the context of a recently proposed method, the LoweAndersen thermostat for interface fluid collisions (LA-IFC, ref 17). It is a modified version of the Lowe-Andersen (LA) thermostat. ${ }^{51}$ The LA-IFC was originally introduced for gas diffusion in the cylindrical pores of carbon nanotubes (CNTs). Gas molecules are not thermalized by random collisions with other gas molecules anymore (basic idea of LA thermostat) but by gas-wall collisions. The LA-IFC treats these collisions, that occur in flexible-lattice simulations deterministically, stochastically in a rigid-framework simulation. Thus, the LA-IFC method is capable of reproducing the main feature of gas diffusion in flexible CNTs and, at the same time, of being very efficient from a computational point of view. Diffusivities calculated with 

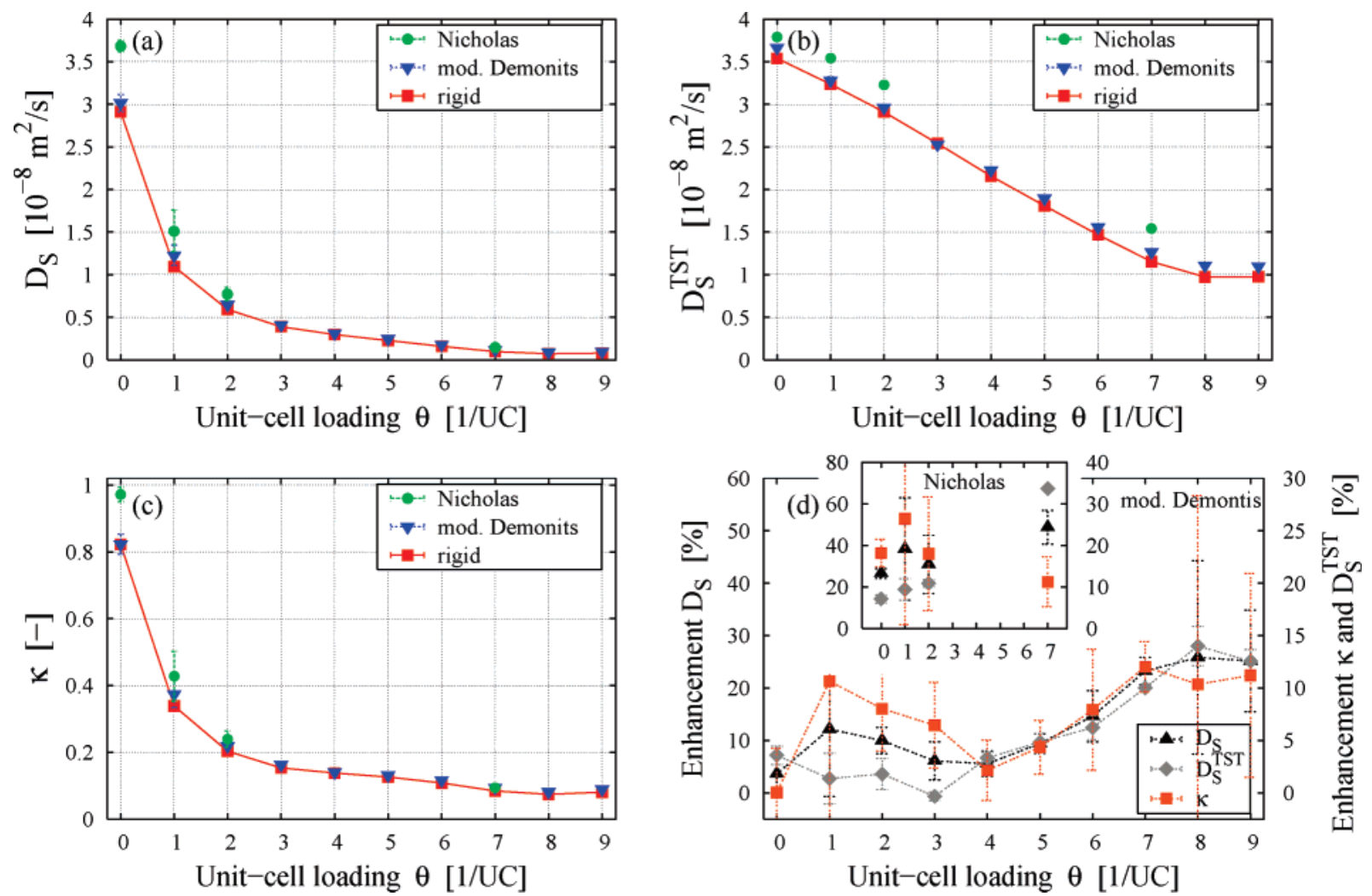

Figure 7. (a,b,c): Self-diffusion coefficient ( $D_{\mathrm{S}}$ top left), TST diffusivity ( $D_{\mathrm{S}}^{\mathrm{TST}}$ top right), and dynamical correction factor $(\kappa$ bottom left) as functions of unit-cell loading, $\theta$, for different framework models. (d): Enhancement factors $\left[\left(A^{\mathrm{FLEX}}-A^{\mathrm{RIGID}}\right) / A^{\mathrm{RIGID}}\right]$ for diffusion quantities $D_{\mathrm{S}}$ on the left $y$ axis and $D_{\mathrm{S}}^{\mathrm{TST}}, \kappa$ on the right $y$ axis as functions of unit-cell loading for both host models (results for Nicholas model in inset). Note that $\kappa$ is obtained from $D_{\mathrm{S}}$ and $D_{\mathrm{S}}^{\mathrm{TST}}$ via eq 13 and that the errors presented for $\kappa$ and the enhancement factors are estimated by Gaussian error propagation. All diagrams: $\mathrm{CH}_{4}$ in $\mathrm{AFI}$ at $300 \mathrm{~K}$.

flexible CNTs are, because of the faster velocity decorrelation, smaller in comparison to diffusivities obtained with rigid CNTs. The parameters for the thermostat that introduces the extradecorrelation are chosen such that it exactly compensates the rigid-framework assumption; that is, collision parameters are to be computed on the basis of VACFs and heating curves of the gas molecules confined in a flexible CNT. For methane in AFI, we find, however, that the "flexible" VACF decreases slower than the "rigid" one. Hence, the LA-IFC cannot be used to mimic the flexibility influence in the present case, because it enhances velocity decorrelation.

\section{Results}

In this section, self-diffusion coefficients obtained from rigidand flexible-framework simulations are presented. We depict free-energy profiles and make use of the concepts of dcTST for understanding the influence of flexible lattices. We follow this route because dcTST is proven to capture the main phenomena of gas diffusion in different types of zeolites correctly, qualitatively, and quantitatively. $22,26,27,48,49$

A. Methane in AFI. In Figure 7, self-diffusion coefficients (top left), ideal TST diffusvities (top right), and dynamical correction factors (bottom left) are shown as functions of loading and lattice model. By means of this diagram, the change in the diffusivity in consequence of the use of flexibility can be compared. Moreover, it gives rise to changes in the free-energy barriers (TST diffusivity) and the interaction of the gas particles (dynamical correction factor). Note that the necessity of the last diagram will become clear in the upcoming discussion.

The self-diffusion coefficient of methane in a rigid AFI framework quickly decreases with loading. Beerdsen et al. ${ }^{49}$ studied the same system with the same set of parameters, only their simulation boxes were longer (eight unit cells in the pore direction). While our results for the TST diffusivities are in good agreement with theirs over the whole loading range, $D_{\mathrm{S}}$ and $\kappa$ differ slightly at zero loading and for $0<\theta \leq 3 / \mathrm{UC}$. This can be attributed to the system size effects explained in section III. The general dependences of loading on $D_{\mathrm{S}}, D_{\mathrm{S}}^{\mathrm{TST}}$, and $\kappa$ are observable also for flexible frameworks. Values for all three quantities obtained from flexible-lattice simulations are, however, always larger than the corresponding rigid-simulation values (except for $D_{\mathrm{S}}^{\mathrm{TST}}$ modified Demontis model at $3 / \mathrm{UC}$ ). Using the Nicholas force field yields consistently the largest value at a given loading.

The larger TST diffusivities are, of course, directly linked to reduced free-energy barriers in the case of flexible lattices. The barriers appear because the zeolite pores are not perfectly smooth but corrugated. There are parts along the pore axis that are narrower (windows) and others that are broader (cages). Hence, the probability distribution of finding a molecule somewhere along the pore axis is not uniform. The wells of the barriers correspond to the location of the cages; the maxima to the windows. Because of the choice of the methane model (no charges considered) and the disregard of aluminum atoms inside the framework, high-energetic adsorption of the gas is not observed; that is, the enthalpy contribution to $F$ is rather small. Therefore, the barriers follow from the structure of the pore and are mainly entropic barriers ( $-T S$ dominating contribution to $F$ ). Clearly, as soon as charges are involved, for example, water or carbon dioxide, the situation will change.

In Figure 8, the normalized free energy $\beta F$ is plotted as a function of normalized reaction coordinate $q / l_{z}^{\text {unitell }}$ for three 

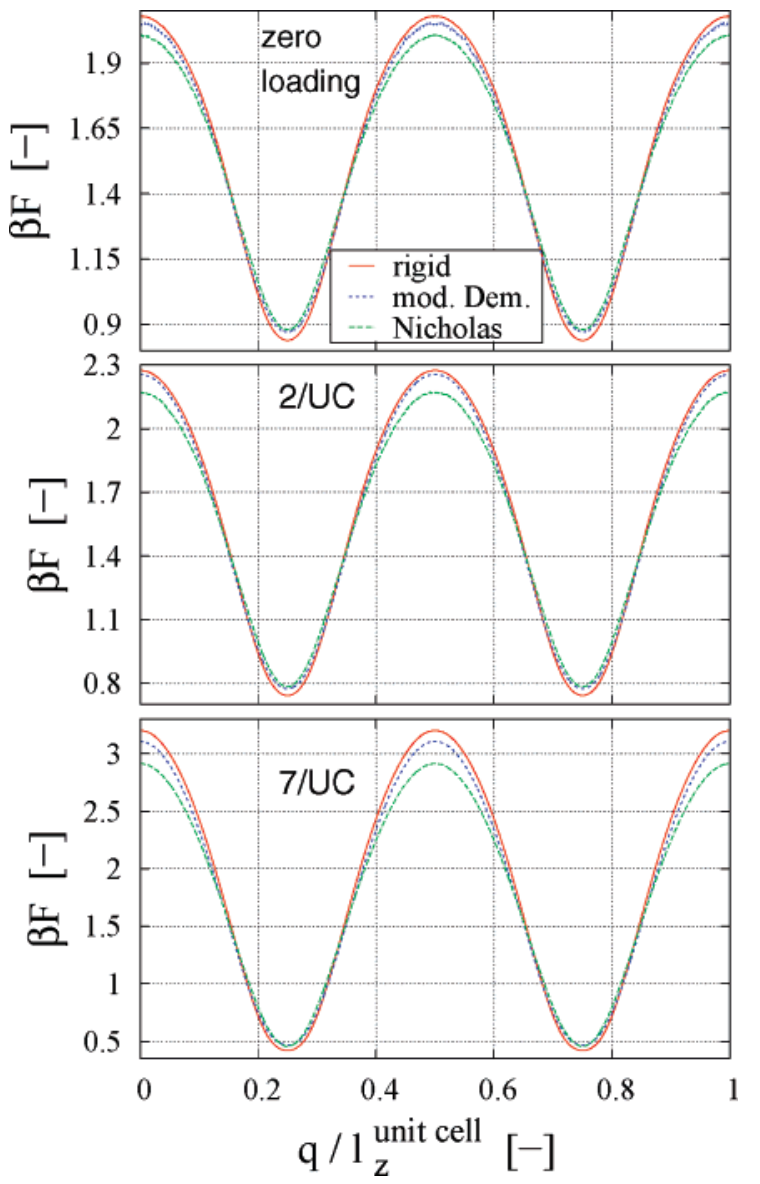

Figure 8. Normalized free energy, $\beta F$, as a function of normalized reaction coordinate, $q / l_{z}^{\text {unitcell }}$, for zero loading as well as $\theta=2$, and 7 molecules/unit cell; $\mathrm{CH}_{4}$ in $\mathrm{AFI}$ at $300 \mathrm{~K}$.

different loadings using all three framework models studied. The net barriers increase with loading, $\theta$, which is the reason for the decrease of $D_{\mathrm{S}}^{\mathrm{TST}}$. At a given loading, the barrier obtained with a rigid lattice is always larger than the one using the modified Demontis model, which is in turn always larger than the one using the Nicholas model. The reduction of the net barriers is strongest at high loading. However, the reduction of $\Delta F$ does not lead to such a large increase in the TST diffusivity that the overall diffusive enhancement is entirely captured by this. Thus, reduced free-energy barriers are not the only reason why framework flexibility leads to diffusive enhancement of the fluid molecules. The dynamical correction factor $\kappa$ is also affected considerably such that successful hops are favored by the use of flexible frameworks.

Since all three quantities decrease rapidly with increasing loading, differences between flexible- and rigid-lattice values are barely visible at high loading. With help of the enhancement diagram (Figure 7 bottom right), we can sort out the dependence of loading itself, while, on a relative base, discussing the deviations between rigid- and flexible-lattice quantities as these vary with loading. As can be seen from it, an enhancement in the diffusion coefficient is most pronounced at high loading. Over a broad loading regime (0/UC $\leq \theta \leq 6 / \mathrm{UC})$, the modified Demontis model results in comparably moderate diffusive enhancement $(4 \ldots 15 \%)$. While at zero loading, this is entirely caused by reduced free-energy barriers, the decrease of interparticle collisions, that is, $\kappa$ is larger from flexible- than from rigid-lattice simulations, has a much larger influence on diffusive enhancement for $1 / \mathrm{UC} \leq \theta \leq 3 / \mathrm{UC}$. At intermediate and high loadings, this changes, and both effects have a similar impact.

The results obtained with the Nicholas force field agree qualitatively with those of the modified Demontis model. However, the magnitudes of enhancement of the two models differ considerably. The diffusive enhancement using the Nicholas model is twice, three times, or even seven times the enhancement obtained with the Demontis model for 7/UC, 1 and 2/UC, and zero loading, respectively. This shows clearly that the Nicholas model yields a distinct enhancement over the whole loading regime in AFI. Furthermore, at zero loading, $\kappa$ is also altered significantly, and reduced free-energy barriers have a more pronounced impact on $D_{\mathrm{S}}$ enhancement at high loading in comparison with the results of the modified Demontis model.

From Table 1, it can be seen that the extent of the flexibility influence in MFI depends also on loading. At low and high loading, zero loading to 5/UC and 12/UC, respectively, an enhancement of the diffusion coefficient by introducing lattice flexibility is obtained that is always larger than $20 \%$. At $8 / \mathrm{UC}$, the enhancement shows, however, a considerably smaller value $(12 \%)$. Thus, a rather complex relation between diffusive enhancement in consequence of the use of flexible lattices and loading is observed which is in agreement with our results. Note that Bandyopadhyay and Yashonath ${ }^{7}$ applied different loadings for comparison of rigid- and flexible-lattice diffusivities. This causes an underestimation of the enhancement because $D_{\mathrm{S}}$ is a monotonically decreasing function of loading for methane in $\mathrm{MFI}^{22}$ and the loading applied in the flexible simulation was larger than in the rigid one.

B. Comparison of $\mathrm{CH}_{4}$ in AFI, LTL, and MTW. To test whether diffusive enhancement is a general phenomenon for one-dimensional zeolite pores, rigid- and flexible-framework MD simulations of methane confined in LTL and MTW at three loadings (zero loading, $\theta=2 / \mathrm{UC}$, and $\theta=7 / \mathrm{UC}$ ) are performed. The results are presented in Table 5 and Figure 9. Rather than discussing the absolute values, we turn our attention again to relative enhancement values. This is because $D_{\mathrm{S}}$ and $\kappa$ decrease even more rapidly for MTW than for AFI, and the enhancement values make a direct comparison of the extent of the flexibility influence between the three materials visually easier.

The influence of framework flexibility on the self-diffusivity is qualitatively similar for AFI and LTL because we observe the smallest enhancement at zero loading and the most

TABLE 5: Diffusion Quantities $D_{\mathrm{S}}, D_{\mathrm{S}}^{\mathrm{TST}}$, and $\kappa$ for LTL and MTW Using Different Framework Models

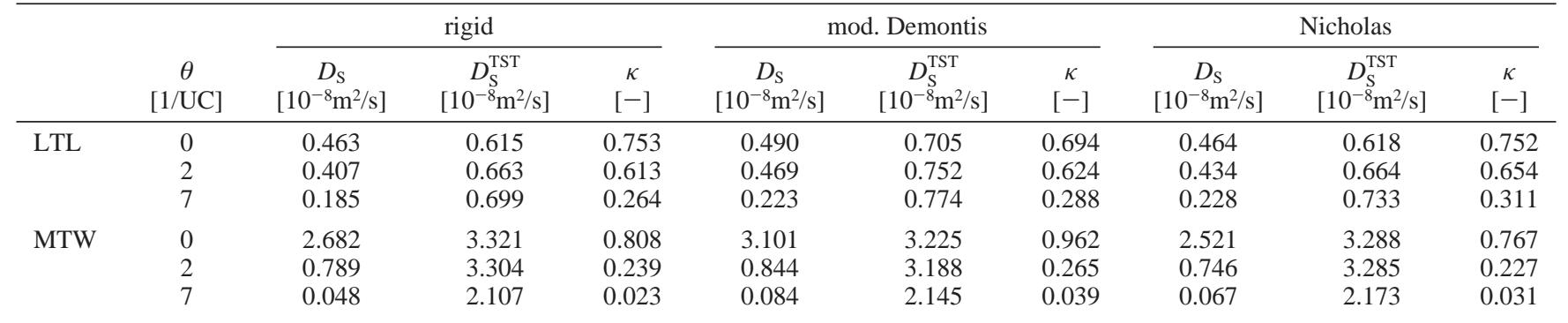


Diffusion coefficient
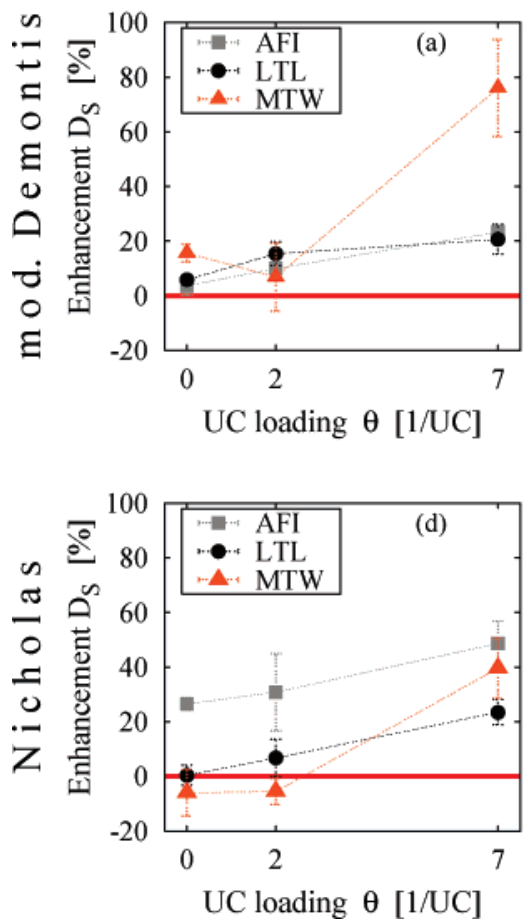

TST diffusivity
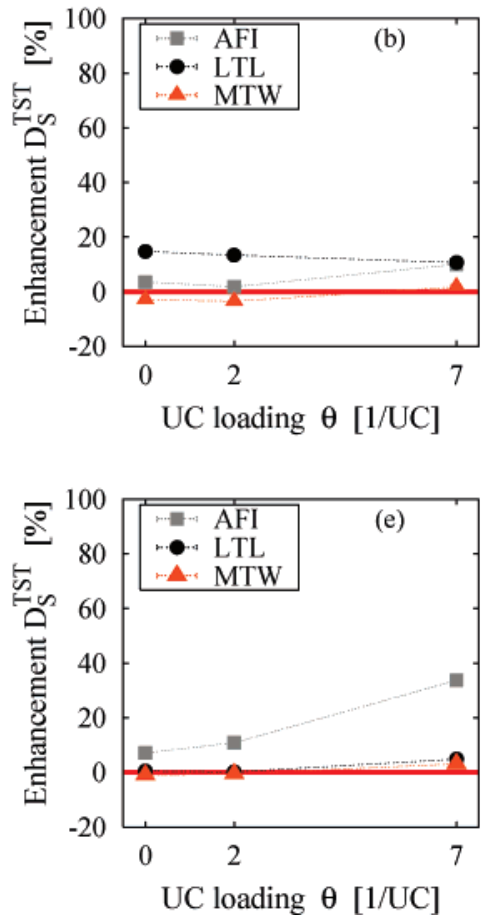

\section{Correction factor}
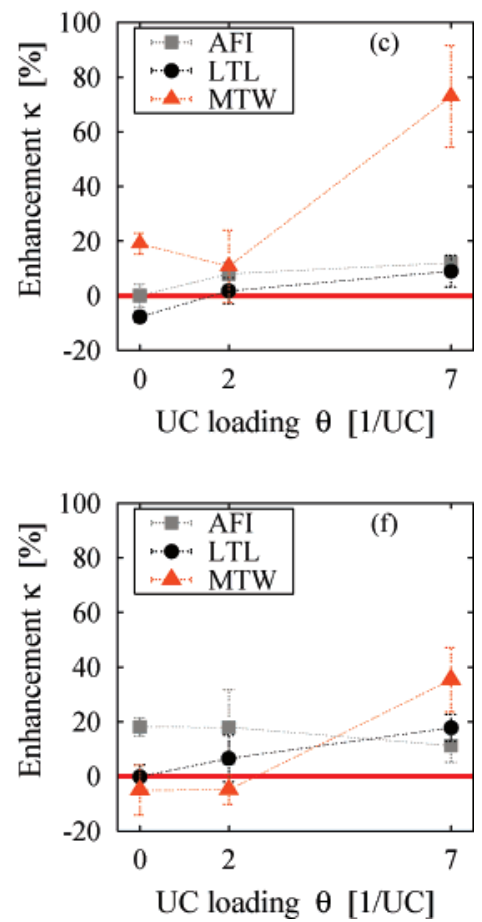

Figure 9. Enhancement factors for self-diffusion coefficient $\left(D_{\mathrm{S}}\right.$ left), TST diffusivity $\left(D_{\mathrm{S}}^{\mathrm{TST}}\right.$ middle), and dynamical correction factor $(\kappa$ right $)$ as functions of unit-cell loading, $\theta$, for AFI, LTL, and MTW (modified Demontis model top, Nicholas model bottom). Note that the errors presented are estimated by Gaussian error propagation.

pronounced enhancement at high loading which is irrespective of the model; see Figure 9a,d. Particularly, for the modified Demontis model, the results coincide for the two materials. However, the Nicholas model yields less pronounced diffusive enhancement in LTL than in AFI, and in addition, we observe negative enhancement at zero loading. The dependence of loading on the enhancement of the TST diffusivity and the associated reduction of free-energy barriers differs very much for AFI and LTL. The Nicholas force field, for low loadings, has hardly any influence on the barrier in LTL. By contrast, the Demontis model yields substantially smaller barriers than the rigid simulations such that the correction factor at zero loading is considerably smaller than the one from rigid simulations. Furthermore, a declining influence of barrier reduction is observed for the Demontis model used in LTL whereas the largest free-energy reduction is observed always at high loading for all of the other cases. At high loading, $D_{\mathrm{S}}^{\mathrm{FLEX}}, D_{\mathrm{S}}^{\mathrm{TST}, \mathrm{FLEX}}$, and $\kappa^{\text {FLEX }}$ are, nonetheless, always larger than the respective rigid values for both materials and both host models.

The results for the MTW zeolite, which has a much smaller window diameter than the other two structures, differ significantly from the results for AFI and LTL. Although we find again more pronounced diffusive enhancement at high loading for both models, the Nicholas model, in contrast to the Demontis force field, yields diffusivities that are nonnegligibly smaller than the rigid values at low loadings. The Demontis model does, furthermore, not yield monotonically increasing enhancement in $D_{\mathrm{S}}$, as observed for AFI and LTL. However, we have seen that diffusive enhancement is not necessarily a monotonically increasing function of loading (consider the AFI data presented in section IV.A and the MFI data from literature). Free-energy barriers do not decrease at low loadings, and compared to the influence flexibility exerts on the behavior of $\kappa$, they always have a smaller influence on $D_{\mathrm{S}}$. As this is observed for both models in MTW, it may be concluded that, if the net barrier observed for a given structure is, on an absolute base, very small, which is the case for MTW in comparison to AFI and LTL, and $\kappa$ has, hence, a very large impact on the diffusivity, flexibility will not yield considerable reduction of the barriers at all.

In essence the results of the comparison shows that diffusive enhancement is not a general behavior of flexible zeolite lattices and that it matters how the flexibility is modeled, that is, which force field is chosen. Moreover, a given model does not necessarily affect the diffusion mechanisms of gas molecules in different zeolites in the same way.

C. Window-Area Distribution. Free-energy barriers that impede the diffusion of small, uncharged gas molecules in zeolites are mostly entropic barriers. It takes some time until the molecule finds its way from one cage through the bottleneck (window) to an adjacent cage which is due to spacious motion in the cage rather than high-energetic adsorption. Therefore, it is generally believed that flexible frameworks that yield smaller barriers lead to temporary broadening of the pore windows, a phenomenon that is also known as the "breathing window" effect. ${ }^{3}$ However, it was also argued that the window does not only broaden but also contracts such that, effectively, no influence of the window motion should be observable. ${ }^{5}$ For this reason, the motion of the window atoms is studied in this section in order to shed more light into the mechanism of barrier reduction. Since the reduction of free-energy barriers does not have a crucial influence on self-diffusion in MTW, we focus on the AFI and LTL structures.

To investigate their motion, we compute area distributions of the windows. The area of a given 12-ring is computed by approximating it as a dodecagon. The crystal structures show that these 12-rings are not regular dodecagons (sides of equal length and angles of equal measure), neither in AFI nor in LTL. The dodecagons consist of two regular hexagons that exhibit 

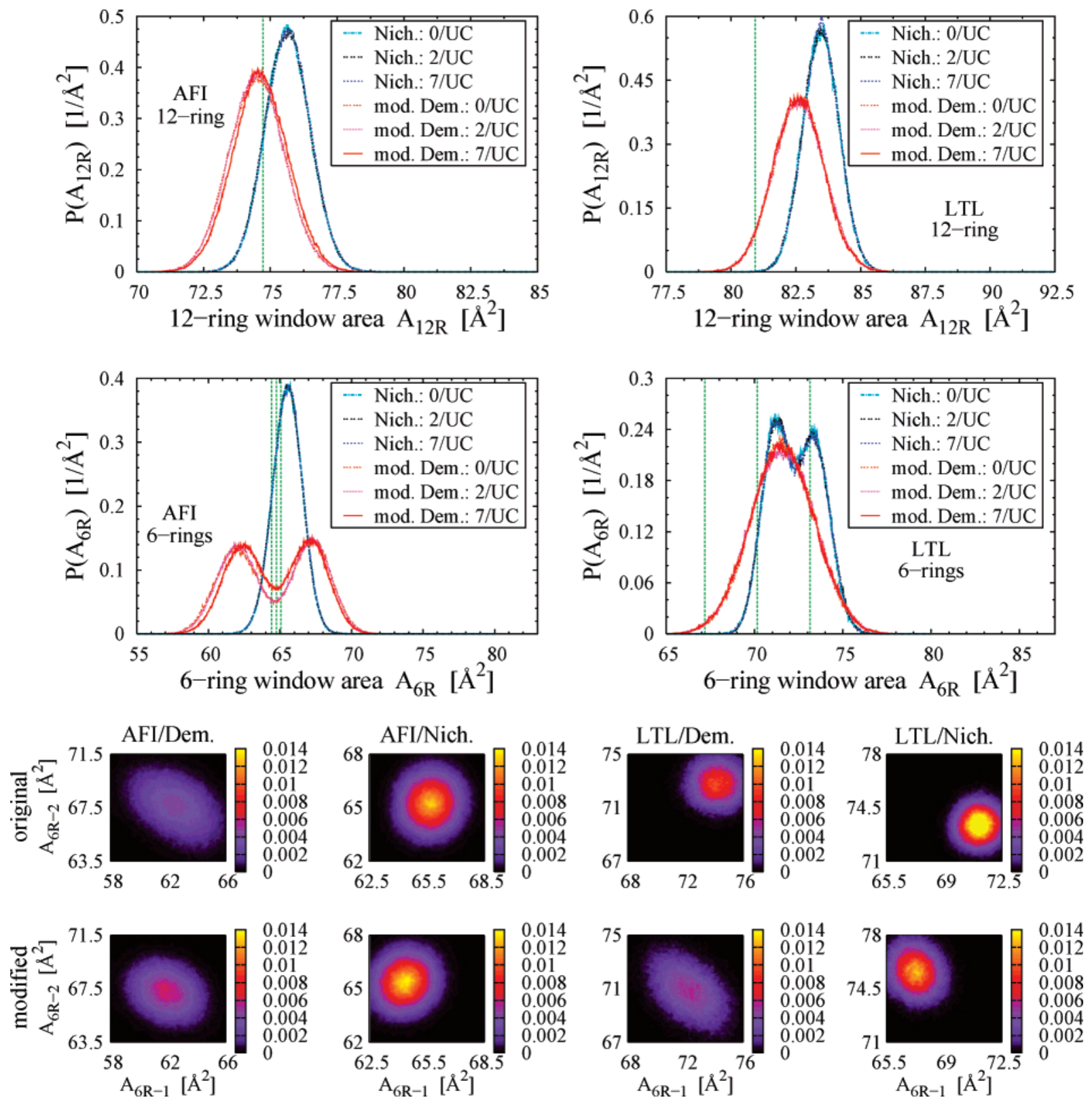

Figure 10. Top and middle: Probability density distributions, $P$, as functions of window area of the 12-ring $A_{12 \mathrm{R}}$ (top) and of the 6-ring $A_{6 \mathrm{R}}$ (middle) for AFI (left) and LTL (right); different loadings and different framework models. The vertical lines represent areas obtained from the crystal structure, whereby the two outer lines in the 6-ring area distributions represent the areas of 6-ring 1 and 6-ring 2, respectively, and the inner line represents the average of both 6-ring areas. Bottom: Probability density distributions as functions of simultaneously observed pairs of 6-ring 1 area $\left(A_{6 \mathrm{R}-1}\right.$ on the $x$ axis) and 6-ring 2 area $\left(A_{6 \mathrm{R}-2}\right.$ on the $y$ axis) for different materials and host models at zero loading (projection in the $A_{6 \mathrm{R}-1}$ $-A_{6 \mathrm{R}-2}$ plane). All diagrams for $\mathrm{CH}_{4}$ at $300 \mathrm{~K}$.

slightly different areas. Hereby, hexagon 1 is obtained by labeling the atoms of the 12-ring clockwise from 1 to 12 , and then draw a hexagon with vertices located at atoms labeled by an even number; hexagon 2 is, hence, obtained with atoms that are labeled by uneven numbers. The difference between the two hexagon areas $\left(\Delta A=A_{6 \mathrm{R}-2}-A_{6 \mathrm{R}-1}=0.327 \AA^{2}\right)$ is marginal in relation to the mean area of both hexagons $(\triangle A / \bar{A}=\Delta A$ / $\left.\left(A_{6 \mathrm{R}-2}+A_{6 \mathrm{R}-1}\right) / 2=0.5 \%\right)$ in the case of AFI, but it is relatively large for LTL $\left(\Delta A=2.980 \AA^{2}, \Delta A / \bar{\AA}=4.3 \%\right)$. In the first place, the motion of the 6-rings does not seem to have any significance for the passage of gas molecules. Our analysis shows, however, that the behavior of the two 6-rings of a given window type is an appropriate indicator for whether or not the structure of a window is described realistically with a given model. Therefore, probability distributions of both ring types, 12- and 6-ring, are computed. The results of the window-area sampling are shown in Figure 10. Note that, in the following, we label the hexagon that exhibits the smaller area in the crystal structure "6-ring 1", and we label the one having a larger area in the crystal structure "6-ring 2".

All 12-ring area distributions are single-peaked, symmetric, and show similar shapes for both materials. However, the distributions obtained with the modified Demontis model are always broader than the ones obtained with the Nicholas model. This indicates that the zeolite atoms modeled by the modified Demontis force field possess larger flexibility. Moreover, the modified Demontis distributions exhibit smaller average values than the distributions of the Nicholas model. Thus, on an average, the window broadens less than with the Nicholas model. The relative differences between the average areas of the two models, that is, $\left(\overline{\mathrm{A}}^{\text {Nicholas }}-\overline{\mathrm{A}}^{\text {Demonits }}\right) / \overline{\mathrm{A}}^{\text {Demonits }}$, are, however, similar for AFI (1.6\%) and LTL (1.0\%). Generally, 
TABLE 6: Relative Deviation of Average 12-ring Window Area from the Reference (Crystal Structure) Area, Order of 6-rings, Relative Deviation of the 6-ring Area Gap $\left(\Delta A_{6 \mathrm{R}}=A_{6 \mathrm{R}-2}-A_{6 \mathrm{R}-1}\right)$, and Relative Reduction of Free-Energy Barrier (if Available) for Different Materials and Models at the Limit of Infinite Dilution ${ }^{a}$

\begin{tabular}{|c|c|c|c|c|c|}
\hline material & model & $\left(\bar{A}_{12 \mathrm{R}}^{\mathrm{F}}-A_{12 \mathrm{R}}^{\mathrm{C}}\right) / A_{12 \mathrm{R}}^{\mathrm{C}}[\%]$ & $\bar{A}_{6 \mathrm{R}-1}^{\mathrm{F}}<\bar{A}_{6 \mathrm{R}-2}^{\mathrm{F}}$ & $\left(\left|\Delta \bar{A}_{6 \mathrm{R}}^{\mathrm{F}}\right|-\Delta A_{6 \mathrm{R}}^{\mathrm{C}}\right) / \bar{A}_{6 \mathrm{R}}^{\mathrm{C}}[\%]$ & $\left(\Delta F^{\mathrm{F}}-\Delta F^{\mathrm{R}}\right) / \Delta F^{\mathrm{R}}[\%]$ \\
\hline AFI & Demontis & 0.47 & yes & 664.8 & \\
\hline AFI & mod. Demontis & -0.32 & yes & 708.1 & 3.6 \\
\hline $\mathrm{AFI}$ & Nicholas & 1.22 & no & -32.1 & 7.1 \\
\hline AFI & mod. Nicholas & 0.25 & yes & 52.8 & 3.0 \\
\hline LTL & Demontis & 4.93 & no & -76.4 & \\
\hline LTL & mod. Demontis & 2.09 & no & -76.3 & 14.7 \\
\hline LTL & Nicholas & 3.13 & yes & -62.6 & 0.5 \\
\hline LTL & mod. Nicholas & 1.81 & yes & 39.9 & \\
\hline
\end{tabular}

${ }^{a}$ Superscript $\mathrm{C}$ stands for a crystal structure value; F and $\mathrm{R}$ stand for values from flexible and rigid-lattice simulations, respectively; subscript $12 \mathrm{R}$ is related to the 12-ring; and $6 \mathrm{R}$ is related to the 6-ring(s).

increasing the loading does not change the distributions significantly. Only the distribution obtained at a loading of 7/UC in AFI using the modified Demontis model varies slightly from the corresponding two other distributions. When this model is used for AFI it can be, hence, regarded as less stiff than the Nicholas model with respect to maintaining the behavior of the window-atoms' motion with increasing gas pressure.

The modified Demontis model used in AFI yields an average area which is very close to the reference area obtained from the crystal structure. This was expected, because individiual equilibrium lengths for the potential terms are taken from the crystal structure for this model, as was explained in section II.A. This more rigorously keeps the zeolite atoms at their crystallographic positions such that the average 12-ring area was also expected to be close to the reference area. Surprisingly, the model does not reproduce the reference area in LTL. The Nicholas model, not incorporating individual, but constant equilibrium lengths and angles, yields average areas that are larger than the reference area for both materials.

The 6-ring area distributions (Figure 10 middle) comprise both 6-rings; that is, they are the superpositions of the 6-ring 1 and 6-ring 2 area distributions for a given material, model, and loading. In contrast to the 12-ring area distributions, the overall 6-ring area distributions are not necessarily single-peaked and symmetric. The distributions obtained with the modified Demontis force field in AFI show two peaks. Thus, the structure of the window is artificially changed by this host model because the peaks indicate that the difference between the average area of 6-ring 1 and 6-ring 2 (in the following called 6-ring gap) are far apart from each other. This is not observed in the crystal structure (outer verticle lines) where the 6-ring gap is small. From this perspective, the Nicholas model seems to be more realistic because the 6-ring gap is similar to the one observed in the crystal structure. In LTL, the crystal structure shows an appreciably large 6-ring gap which is not reproduced well by either of the models.

In Figure 10 bottom, probability distributions of observing simultaneously a given 6-ring 1 area and 6-ring 2 area are presented for AFI and LTL at the limit of infinite dilution. The $x$ axis represents the area of 6-ring 1, the one that exhibits the smaller area in the cyrstal structure; the $y$ axis represents thus the (supposedly) larger 6-ring 2. To investigate the influence of modifying a given framework model by implementing equilibrium values from the crystal structure, additional distributions are computed including the original Demontis and the "modified Nicholas" model. There are three cases where the two 6-rings switch "roles", that is, $\bar{A}_{6 \mathrm{R}-1}>\bar{A}_{6 \mathrm{R}-2}$, namely, AFI/ Nicholas, LTL/Demontis, and LTL/modified Demontis. For AFI, it can be seen that the modification enables the original Nicholas model to reproduce the right order (6-ring 1 smaller on an average than 6-ring 2). However, for the LTL/Demontis case, this does not hold because, even with the modification, the 6-ring 1 is on an average still larger than the 6-ring 2. As was argued before, the reason might be the smaller "stiffness" of this model. The modification that may be interpreted as additional constraints is not yet constraining the zeolite atoms strong enough to yield qualitatively correct results (order of the 6-rings). This indicates an incorrect description of the motion of the zeolite atoms. Finally, it can be seen from Table 6 which summarizes the most important details of the area distributions that the modified Nicholas model yields the best agreement with the respective details of the crystal structure for both materials but particularily for AFI.

\section{Conclusions}

Table 1 indicates that the choice of the host model is important for the extent of the enhancement of the diffusion coefficient induced by introducing a flexible zeolite. In this work, we have observed that the way in which flexibility is modeled is crucial to the results not only in a quantitive way but, more surprisingly, the two models influence the diffusivity in qualitatively different ways. In MTW, we observe very little influence of flexibility on the free energy barriers, but because of differences in recrossing, the Nicholas model yields smaller diffusivities, and the modified Demontis model larger diffusivities than the rigid lattice at low loading. However, both models predict considerably larger diffusion coefficient than the rigid-lattice simulations at high loading which is also seen for the other two zeolites.

From a free energy point of view, lattice vibration generally reduces the barrier for diffusion because the average window is larger than the corresponding window for a rigid lattice. A larger average window normally results in a larger diffusion coefficient, for example, LTL with the Demontis model or AFI at high loading. This is, however, not generally true as zeolite flexibility can result in a much lower recrossing rate resulting in a decrease of the diffusion coefficient, for example, LTL model with the Nicholas model. This shows that lattice vibration influences the diffusion coefficient in different ways and that the underlying dynamics, as expressed by the window-area distribution, of both models can be very different.

Finally, we would like to address the question whether a flexible zeolite gives a better prediction of the diffusion coefficient compared with a rigid model. One can argue that a flexible zeolite is a more realistic description of a true zeolite such that the answer must be "of course". However, the results presented in this work show that one should be careful. Simply taking any force field to model a flexible zeolite may give a prediction of a diffusion coefficient that, depending on the system, might be worse than a prediction using a rigid lattice. 
This statement is based on our observation, that, to the best of our knowledge, the dynamics of the zeolite framework is not yet sufficiently understood and that there are no clear criteria on which force field gives the most realistic description. Of course, first principle calculations have the potential to improve the existing zeolite models, and it can be speculated that within a finite number of years it will be possible to use Car-Parrinello MD calculations or the like for diffusion studies in zeolites. However, these techniques are at present still limited by insufficient computer performances. Ultimately, it is important to mention that, for the systems we have studied here, the effect of flexibility is not extreme in the sense that the molecules do not move in a rigid zeolite, that is, diffusion of bulky molecules which is possible only because of activation from vibrating zeolite atoms. Hence, we are probing much more subtle effects than what is intended with the "breathing window" in the experimental work.

Acknowledgment. This paper is dedicated to Professor Keith E. Gubbins on the occasion of his 70th birthday. This work was supported by the Deutsche Forschungsgemeinschaft (DFG, priority program SPP 1155), the EC through the Marie Curie EST project "EuroSim" (MEST-CT-2005-020491), and the EXT project "BiMaMoSi" (MEXT-CT-2005-023311).

\section{References and Notes}

(1) Kärger, J.; Ruthven, D. M. Diffusion in Zeolites and Other Microporous Solids; John Wiley \& Sons Inc: New York, 1992.

(2) Barrer, R. M.; Vaughan, D. E. W. J. Phys. Chem. Solids 1971, 32, 731-743.

(3) Deem, M. W.; Newsam, J. M.; Creighton, J. A. J. Am. Chem. Soc. 1992, 114, 7198-7207.

(4) Smirnov, K. S.; Bougeard, D. Zeolites 1994, 14, 203-207.

(5) Fritzsche, S.; Wolfsberg, M.; Haberlandt, R.; Demontis, P.; Suffritti, G. B.; Tilocca, A. Chem. Phys. Lett. 1998, 296, 253-258.

(6) Demontis, P.; Suffritti, G. B.; Mura, P. Chem. Phys. Lett. 1992, $191,553-560$. 4292.

(7) Bandyopadhyay, S.; Yashonath, S. J. Phys. Chem. 1995, 99, 4286-

(8) Smirnov, K. S. Chem. Phys. Lett. 1994, 229, 250-256.

(9) Fritzsche, S.; Wolfsberg, M.; Haberlandt, R. Chem. Phys. 2001, $289,321-333$.

(10) Demontis, P.; Suffritti, G. B.; Fois, E. S.; Quartieri, S. J. Phys. Chem. 1992, 96, 1482-1490.

(11) Thomson, K. T.; McCormick, A. V.; Davis, H. T. J. Chem. Phys. 2000, 112, 3345-3350.

(12) Leroy, F.; Rousseau, B.; Fuchs, A. H. Phys. Chem. Chem. Phys. 2004, 6, 775-783.

(13) Demontis, P.; Suffritti, G. B.; Quartieri, S.; Fois, E. S.; Gamba, A. J. Phys. Chem. 1988, 92, 867-871.

(14) Demontis, P.; Suffritti, G. B.; Quartieri, S.; Fois, E. S.; Gamba, A. Zeolites 1987, 7, 522-527.

(15) Kantola, J.-H.; Vaara, J.; Rantala, T. T.; Jokisaari, J. J. Chem. Phys. 1997, 107, 6470-6478.

(16) Demontis, P.; González, J. G.; Suffritti, G. B.; Tilocca, A. J. Am. Chem. Soc. 2001, 123, 5069-5074.
(17) Jakobtorweihen, S.; Verbeek, M. G.; Lowe, C. P.; Keil, F. J.; Smit, B. Phys. Rev. Lett. 2005, 95, 044501.

(18) Jakobtorweihen, S.; Lowe, C. P.; Keil, F. J.; Smit, B. J. Chem. Phys. 2006, 124, 154706.

(19) Vlugt, T. J. H.; Schenk, M. J. Phys. Chem. B 2002, 106, 1275712763.

(20) Smirnov, K. S.; Bougeard, D. J. Phys. Chem. 1993, 97, 94349440.

(21) Baerlocher, C.; Meier, W. M.; Olson, D. H. Atlas of Zeolite Framework Types; Elsevier Science B. V.: Amsterdam, 2001.

(22) Beerdsen, E.; Dubbeldam, D.; Smit, B. J. Phys. Chem. B 2006, 110, 22754-22772.

(23) Qiu, S. L.; Pang, W. Q.; Kessler, H.; Guth, J. L. Zeolites 1989, 9 , 440-444.

(24) Fyfe, C. A.; Gies, H.; Kokotailo, G. T.; Marler, B.; Cox, D. E. J. Phys. Chem. 1990, 94, 3718-3721.

(25) Barrer, R. M.; Villiger, H. Z. Kristallogr. 1969, 128, 352-370.

(26) Beerdsen, E.; Smit, B.; Dubbeldam, D. Phys. Rev. Lett. 2004, 93 , 248301.

(27) Dubbeldam, D.; Beerdsen, E.; Vlugt, T. J. H.; Smit, B. J. Chem. Phys. 2005, 112, 224712.

(28) Dubbeldam, D.; Calero, S.; Vlugt, T. J. H.; Krishna, R.; Maesen, T. L. M.; Beerdsen, E.; Smit, B. Phys. Rev. Lett. 2004, 93, 088302.

(29) Dubbeldam, D.; Calero, S.; Vlugt, T. J. H.; Krishna, R.; Maesen,

T. L. M.; Smit, B. J. Phys. Chem. B 2004, 108, 12301-12313.

(30) Nicholas, J. B.; Hopfinger, A. J.; Trouw, F. R.; Iton, L. E. J. Am. Chem. Soc. 1991, 113, 4792-4800.

(31) Schüring, A.; Auerbach, S. M.; Fritzsche, S.; Haberlandt, R. $J$. Chem. Phys. 2002, 116, 10890-10894.

(32) Kärger, J.; Demontis, P.; Suffritti, G. B.; Tilocca, A. J. Chem. Phys. 1999, $110,1163-1172$

(33) Lopez, A.; Soulard, M.; Guth, J. Zeolites 1990, 10, 134-136.

(34) Bhide, S. Y.; Yashonath, S. J. Chem. Phys. 2002, 116, 2175-2183.

(35) Fritzsche, S.; Osotchan, T.; Schüring, A.; Hannongbua, S.; Kärger, J. Chem. Phys. Lett. 2005, 411, 423-428.

(36) Bezus, A. G.; Kiselev, A. V.; Lopatkin, A. A.; Du, P. Q. J. Chem. Soc., Faraday Trans. 2 1978, 74, 367-379.

(37) Macedonia, M. D.; Maginn, E. J. Mol. Phys. 1999, 96, 1375-1390.

(38) Choudhary, V. R.; Mayadevi, S. Langmuir 1996, 12, 980-986.

(39) Martin, C.; Tosi-Pellenq, N.; Patarin, J.; Coulomb, J. P. Langmuir 1998, 14, 1774-1778.

(40) Maris, T.; Vlugt, T. J. H.; Smit, B. J. Phys. Chem. B 1998, 102, $7183-7189$.

(41) Boutin, A.; Pellenq, R. J. M.; Nicholson, D. Chem. Phys. Lett. 1994, $219,484-490$.

(42) Moon, S. D.; Miyano, Y. Bull. Kor. Chem. Soc. 1997, 18, 291295.

(43) Ndjaka, J. M. B.; Zwanenburg, G.; Smit, B.; Schenk, M. Microporous Mesoporous Mater. 2004, 68, 37-43.

(44) Liu, B.; Smit, B.; Calero, S. J. Phys. Chem. B 2006, 110, 2016620171.

(45) Chandler, D. Introduction to Modern Statistical Mechanics; Oxford University Press: New York, 1987.

(46) Frenkel, D.; Smit, B. Understanding Molecular Simulations, From Algorithms to Applications; Academic Press: San Diego, CA, 2002.

(47) Tepper, H. L.; Hoogenboom, J. P.; van der Vegt, N. F. A.; Briels, W. J. J. Chem. Phys. 1999, 110, 11511-11516.

(48) Beerdsen, E.; Dubbeldam, D.; Smit, B. Phys. Rev. Lett. 2005, 95 164505 .

(49) Beerdsen, E.; Dubbeldam, D.; Smit, B. Phys. Rev. Lett. 2006, 96 , 044501

(50) Martyna, G. J.; Tuckerman, M. E.; Tobias, D. J.; Klein, M. L. Mol. Phys. 1996, 87, 1117-1157.

(51) Lowe, C. P. Europhys. Lett. 1999, 47, 145-151. 